Reprod. Nutr. Dévelop., 1983, 23 (3), 625-640.

\title{
La vitellogénine chez un Crustacé Décapode Natantia, Palaemon serratus Pennant. Mise en évidence, comparaison immunologique avec les vitellines, site de synthèse et rôle des pédoncules oculaires
}

\author{
J.-J. MEUSY, Henriette JUNERA, P. CLEDON, Madeleine MARTIN
}

Laboratoire de Sexualité et Reproduction des Invertébrés, ERA 070409, Université P. et M. Curie, Bât. A, 4 place Jussieu, 75230 Paris Cedex 05.

Summary. Vitellogenin in a decapod crustacean Palaemon serratus. Identification, immunological similarity to vitellin, synthesis and role of the eyestalks.

Vitellogenin, the precursor of vitellin, has been identified by disc-electrophoresis in the haemolymph of vitellogenic females of Palaemon serratus (Crustacea, Decapoda, Natantia). It appeared in the form of two bands of low electrophoretic mobility and was immunologically indistinguishable from vitellin. The two forms of vitellogenin were radioactive 6 to $7 \mathrm{~h}$ after the injection of ${ }^{3} \mathrm{H}$-leucine to vitellogenic females, while no labelling was detected in the ovary. Therefore, contrary to the opinion of some authors, ovarian metabolism was not the cause of the presence of vitellogenin in the haemolymph.

Immunohistochemical analysis revealed a high content of vitellogenin in the lateral and particularly the ventral sub-epidermal fat body of these females. With the exception of ovary and haemolymph, no other organ or tissue was immunoreactive. This result, reported for the first time in a decapod crustacean indicates that the fat body is the site of vitellogenin synthesis.

In non-vitellogenic females, vitellogenin was no longer synthesized and was not found in the haemolymph. Removal of the eyestalks in non-vitellogenic females induced the onset of vitellogenin synthesis as a consequence of the suppression of the inhibitory effect of the gonado-inhibiting hormone (GIH).

\section{Introduction.}

L'existence d'un précurseur sérique des vitellines, la vitellogénine, a été mise en évidence chez plusieurs espèces de Crustacés (voir : Meusy, 1980) et sa synthèse par le tissu adipeux a été démontrée chez un Crustacé Amphipode, Orchestia gammarellus Pallas (Junéra et Croisille, 1980) de même que chez les Isopodes Porcellio dilatatus Brandt (Picaud et Souty, 1980a et b) et /dotea balthica basteri Audoin (Souty et Picaud, 1981). Ainsi, la vitellogenèse " secondaire " (Charniaux-Cotton, 1978) paraît obéir chez ces deux ordres de Crustacés Péracarides à un schéma déjà mis en évidence chez de nombreuses espèces d'Insectes. Cependant, certaines observations en microscopie électronique réali- 
sées chez les Crustacés Décapodes semblent mettre en cause l'existence d'un précurseur extra-ovarien du vitellus (Beams et Kessel, 1962, 1963 ; Kessel, 1968 ; Ganion et Kessel, 1972). La question de l'origine du vitellus secondaire a été soulevée également par les travaux de deux groupes d'auteurs qui ont obtenu le marquage du vitellus (Arcier et Bonami, 1979, chez Palaemon adspersus) ou de certaines sous-unités polypeptidiques de la vitelline (Lui, Sage et $O^{\prime}$ Connor, 1974, Lui et O'Connor, 1976, chez l'Ecrevisse Procambarus sp. ; Lui et O'Connor, 1977, chez le Crabe Pachygrapsus crassipes) en incubant in vitro des ovaires en vitellogenèse en présence d'acides aminés marqués radioactifs. Bien qu'il existe, au moins chez les espèces Palaemon adspersus et Procambarus sp., une protéine sérique non distinguable immunologiquement des vitellines (Arcier et Tournamille, 1974 ; Fyffe et $O^{\prime}$ Connor, 1974), celle-ci pourrait provenir du vitellus au lieu d'en être le précurseur. Lui, Sage et O'Connor (1974) pensent en effet que la présence de cette protéine dans l'hémolymphe serait due à la résorption du vitellus des ovocytes non pondus. Nous avons utilisé une espèce proche de celle étudiée par Arcier et Bonami, Palaemon serratus, et nous avons cherché à savoir s'il existe chez ce Décapode une protéine sérique répondant à la définition de la vitellogénine et, en particulier, synthétisée hors de l'ovaire.

\section{Matériel et méthodes.}

Animaux. - Les Crevettes proviennent de la Station biologique de Roscoff (Nord-Finistère) et sont conservées à $16^{\circ} \mathrm{C}$ dans des aquariums dont le fond est recouvert d'une couche de calcaire coquillier. Elles sont généralement utilisées peu de temps après leur arrivée. Une eau de mer synthétique à $35 \%$ est utilisée (h-w Marinemix, Wiegandt, Allemagne Fédérale).

L'état de l'ovaire est facilement observable au travers de la cuticule. Transparent et incolore durant la phase de repos sexuel, l'ovaire devient en effet vert pâle en début de vitellogenèse secondaire, puis vert foncé et enfin brun-verdâtre en fin de vitellogenèse.

Préparation des échantillons. - L'hémolymphe est prélevée dans la cavité péricardiale par capillarité ou ponction et mélangée volume à volume à une solution de citrate de sodium à $10 \%$ afin d'éviter la coagulation. Les hémocytes sont éliminés par centrifugation (17 $000 \mathrm{~g} / 15 \mathrm{~min})$.

Les ovaires sont pesés et rincés dans du tampon phosphate de sodium $\left(\mathrm{Na}_{2} \mathrm{HPO}_{4} / \mathrm{NaH}_{2} \mathrm{PO}_{4}\right) 0,1 \mathrm{M}$ à pH 7,2. Ils sont homogénéisés au Potter à $5{ }^{\circ} \mathrm{C}$ dans ce tampon à raison de $4 \mathrm{ml}$ de tampon pour $1 \mathrm{~g}$ d'organe frais. Le broyat est ensuite centrifugé à $17000 \mathrm{~g}$ pendant $15 \mathrm{~min}$ et le surnageant est prélevé en évitant d'entraîner les lipides présents en surface.

Electrophorèse en gel de polyacrylamide. - Les électrophorèses sont effectuées en tubes verticaux de $6 \mathrm{~mm}$ de diamètre, selon la méthode de Davis (1964). Les gels de séparation, de $11 \mathrm{~cm}$ de hauteur, contiennent $7 \%$ d'acrylamide et sont surmontés d'un gel de tassement de $1 \mathrm{~cm}$. La migration est effectuée en chambre froide pendant $3 \mathrm{~h} 40(2,5 \mathrm{~mA}$ par tube). Le tampon utilisé pour les cuves est le Tris-glycine à $\mathrm{pH} 8,4$. Le front est visualisé par l'adjonction 
de bleu de bromophénol à l'échantillon. Après fixation, les gels sont colorés par le bleu de Coomassie.

Etude de la synthèse de la vitellogénine au moyen de leucine tritiée. - Les animaux, dont la longueur (rostre-telson) est d'environ $6 \mathrm{~cm}$, reçoivent en début de matinée dans le vaisseau dorsal, au niveau des $2^{\mathrm{e}}$ et $4^{\mathrm{e}}$ segments abdominaux, une injection de $10 \mu \mathrm{l}$ de leucine ${ }^{3} \mathrm{H}\left(\mathrm{L}\left[4-5-{ }^{3} \mathrm{H}\right]\right.$ leucine ; A.S. : $165 \mathrm{Ci} / \mathrm{mmole}$; Radiochemical Centre, Amersham). Afin de vérifier l'absence de rejet, la leucine${ }^{3} \mathrm{H}$ injectée est colorée par le bleu de méthylène. 6 ou 7 heures après l'injection, un prélèvement d'hémolymphe est effectué et mélangé à une solution aqueuse de citrate de $\mathrm{Na}$ à $10 \%$ afin d'éviter la coagulation. Un volume correspondant à $7 \mu l$ d'hémolymphe non diluée est utilisée pour l'électrophorèse.

La zone du gel où migre la vitellogénine (ou les vitellines, dans le cas d'électrophorèse d'extraits d'ovaires) est prélevée et dissoute dans $1 \mathrm{ml} \mathrm{H}_{2} \mathrm{O}_{2}$ à 110 vol. pendant $16 \mathrm{~h}$ à $60^{\circ} \mathrm{C}$. Après refroidissement, $10 \mathrm{ml} \mathrm{d}$ Instagel Packard sont ajoutés. Le comptage est effectué avec un compteur à scintillation liquide Intertechnique après un délai d'au moins $4 \mathrm{~h}$ afin d'éviter la chimiluminescence. Tous les échantillons étant traités de façon identique, le "quenching » est constant (vérification à l'aide du standard externe). Les résultats sont exprimés en cpm par $7 \mu l$ d'hémolymphe.

Protocole d'immunisation et d'absorption. - Deux ovaires en vitellogenèse secondaire, pesant $0,80 \mathrm{~g}$, sont broyés dans $4 \mathrm{ml}$ de tampon phosphate de sodium selon la méthode indiquée précédemment. Après centrifugation, une partie du surnageant est additionnée volume à volume d'adjuvant de Freund complet. Une $1^{\text {re }}$ injection intramusculaire de $1 \mathrm{ml}$ de ce mélange est effectuée à chacun des 2 lapins utilisés. Un mois plus tard, on pratique une $2^{\mathrm{e}}$ injection, intraveineuse, d'un mélange constitué par $0,2 \mathrm{ml}$ de surnageant et $0,8 \mathrm{ml}$ de tampon phosphate de $\mathrm{Na}$. Après un délai supplémentaire de 2 mois, une $3^{e}$ injection intraveineuse est pratiquée $10,05 \mathrm{ml}$ de surnageant $+0,45 \mathrm{ml}$ de tampon phosphate de $\mathrm{Na}$ ). Enfin, 4 mois plus tard, les lapins reçoivent une $4^{\mathrm{e}}$ injection intramusculaire multisite $(0,2 \mathrm{ml}$ de surnageant $+0,3 \mathrm{ml}$ de tampon phosphate de $\mathrm{Na}$ $+0,5 \mathrm{ml}$ d'adjuvant de freund incomplet).

Les sérums prélevés sur chacun des 2 lapins 1 semaine après les $2^{\mathrm{e}}, 3^{\mathrm{e}}$ et $4^{\mathrm{e}}$ injections sont testés contre un extrait d'ovaires en vitellogenèse ( $E O V$ )) et sont réunis.

L'absorption des immunsérums (témoins) est effectuée de 2 façons différentes :

- absorption par de l'hémolymphe, citratée et centrifugée, de femelle en vitellogenèse. Il est tenu compte de la dilution de l'hémolymphe par la solution de citrate de $\mathrm{Na}$ afin que l'absorption soit effectuée volume à volume.

- Absorption par des extraits d'animaux entiers (femelles en vitellogenèse secondaire ou mâles). Les animaux sont broyés à I'Ultra-Turrax dans les mêmes conditions que les ovaires. L'absorption est effectuée volume à volume.

Le mélange immunsérum-antigènes séjourne $2 \mathrm{~h}$ à $42{ }^{\circ} \mathrm{C}$ puis 1 nuit à la température du laboratoire avant centrifugation $(17000 \mathrm{~g} / 20 \mathrm{~min})$ et élimination du culot. 
Les extraits d'ovaires en repos sexuel sont préparés de la même façon que ceux d'ovaires en vitellogenèse.

Techniques immunologiques. - Les double-diffusions radiales sont effectuées en gel d'agarose IBF A-45 à 0,9\% dans du tampon barbital $24,5 \mathrm{mM}$ à pH 8,6 selon la technique d'Ouchterlony (1948).

Les immunoélectrophorèses croisées sont réalisées soit entièrement en gel d'agarose pour les 2 migrations (même gel que précédemment), soit en gel de polyacrylamide pour la $1^{\text {re }}$ migration (selon la technique exposée plus haut) et en gel d'agarose pour la $2^{e}$ migration.

La $1^{\text {re }}$ migration en agarose s'effectue sous une tension de $12 \mathrm{~V} / \mathrm{cm}$ pendant environ 50 min (tampon des cuves : barbital 24,5 mM à pH 8,6 (*)). Le gel d'agarose dans lequel est réalisée la $2^{\mathbf{e}}$ migration, à 90 degrés par rapport à la 1 re , est additionné d'antisérum dirigé contre les extraits d'ovaires en vitellogenèse secondaire, préalablement dialysé pendant 1 nuit contre du tampon barbital $12,5 \mathrm{mM}$ à $\mathrm{pH} 8,6$. Dans le cas d'une immunoélectrophorèse agarose-agarose, la $2^{\mathrm{e}}$ migration s'effectue sous une tension de $12 \mathrm{~V} / \mathrm{cm}$ pendant $17 \mathrm{~h} 30$. Pour une immunoélectrophorèse acrylamide-agarose, la tension utilisée lors de la $2^{\mathrm{e}}$ migration est de $1,5 \mathrm{~V} / \mathrm{cm}$ pendant $72 \mathrm{~h}$.

Techniques immunohistochimiques. - Le céphalothorax et l'abdomen sont séparés et perfusés par une solution de paraformaldéhyde à $1 \%$ dans du tampon phosphate $(50 \mathrm{mM}, \mathrm{pH} \mathrm{7,4)}$ contenant du saccharose (100 mM) selon le protocole décrit lors d'un précédent travail (Croisille et Junéra, 1980).

La vitellogénine est mise en évidence sur coupes histologiques transversales de $7 \mu \mathrm{m}$ d'épaisseur par une technique immunoenzymatique utilisant la peroxydase (Avrameas, 1969). Les coupes sont recouvertes successivement par l'immunserum produit contre les extraits d'ovaires en vitellogenèse secondaire (EOV) de Palaemon serratus (dilution 1/40), des anticorps de mouton antiglobulines de Lapin marqués par la peroxydase (Institut Pasteur Paris) et le réactif de Graham et Karnovsky (1966).

La réaction est améliorée en traitant préalablement les coupes avec un sérum de mouton normal (Institut Pasteur) et en absorbant par un extrait de mâles le sérum anti-EOV.

Divers témoins sont réalisés. Les témoins immunologiques sont obtenus :

- en remplaçant le sérum anti-EOV par du sérum de lapin prélevé avant immunisation et absorbé par un extrait de mâles ;

- en épuisant le sérum anti-EOV par un extrait de $P$. serratus femelle en vitellogenèse ;

- enfin, en omettant le traitement par le sérum anti-EOV.

Les témoins physiologiques retenus sont des mâles et des femelles en repos sexuel qui ne présentent pas de traces de vitellogénine dans I'hémolymphe.

${ }^{*}$ ) Barbital 24,5 mM, Tris $73 \mathrm{mM}$, azide de $\mathrm{Na} 0,2 \mathrm{mM}$, lactate de $\mathrm{Na} 0,36 \mathrm{mM}$. 


\section{Résultats.}

Recherche par électrophorèse en gel de polyacrylamide des protéines sériques impliquées dans la vitellogenèse secondaire. - La comparaison des électrophorégrammes d'hémolymphe de mâles et de femelles en repos sexuel avec ceux de femelles en vitellogenèse secondaire révèle l'existence de 2 constituants liés à la vitellogenèse secondaire (fig. 1). Le premier, de $\operatorname{Rf} 5,65 \pm 0,73$ (VG 1) est plus abondamment représenté que le second (VG 2) de $\operatorname{Rf} 11,97 \pm 0,56$.

La comparaison des électrophorégrammes d'extraits d'ovaires en vitellogenèse primaire (ou " prévitellogenèse $")$ ) avec ceux d'extraits d'ovaires en vitellogenèse secondaire montre que le vitellus secondaire est essentiellement constituè par 2 vitellines, $\vee 1$ et $\vee 2$, de mobilités analogues à celles de VG 1 et VG 2, quoique assez mal définies (fig. 1). Au fur et à mesure que la vitellogenèse secondaire se déroule, V 1 et $\mathrm{V} 2$ deviennent extrêmement abondantes et apparaissent comme les constituants majeurs de l'ovaire.

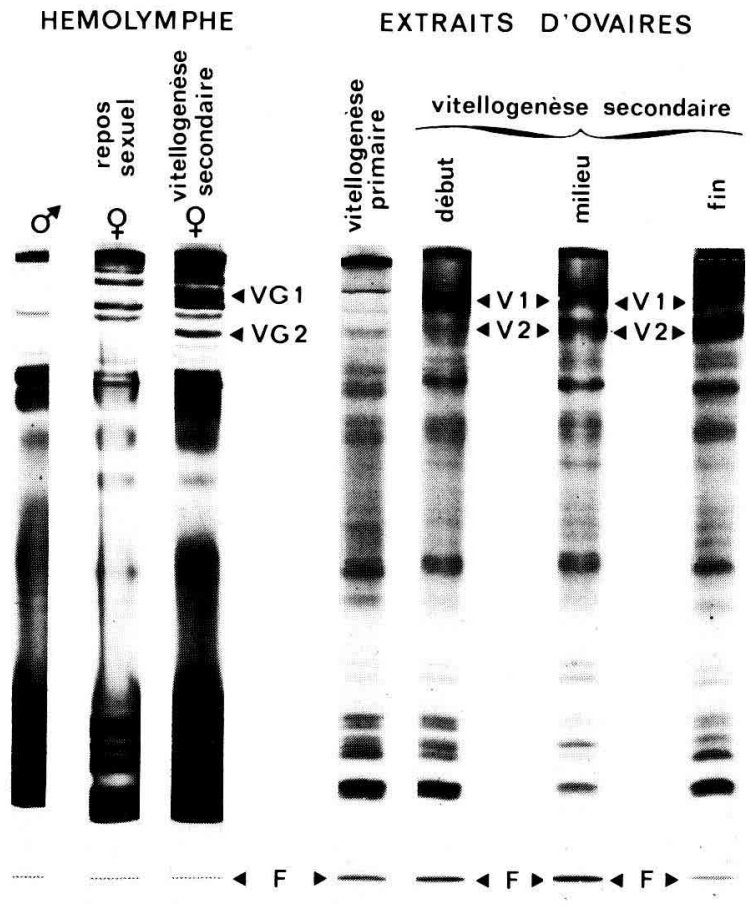

FIG. 1. - Electrophorèses en gel de polyacrylamide d'hémolymphe et d'extraits d'ovaires. $\vee 1$ et V 2 : vitellines 1 et 2 ; VG 1 et VG 2 : vitellogénine apparaissant sous forme de 2 bandes. 
Analyses immunologique et immunoélectrophorétique comparées des constituants de l'hémolymphe et des ovaires des femelles en vitellogenèse secondaire. - En double-diffusion radiale selon Ouchterlony, l'hémolymphe et les extraits d'ovaires (EOV) des femelles en vitellogenèse secondaire donnent, en présence du sérum anti-EOV, 1 ou 2 lignes de précipitation, plus rarement 3. Ces lignes de précipitation sont en rapport avec la vitellogenèse secondaire: elles ne sont observées ni avec l'hémolymphe des mâles ni avec celle des femelles en repos sexuel (fig. 2).

Quel que soit le nombre de lignes de précipitation mises en évidence, aucun éperon n'apparaît jamais entre celles-ci lorsqu'un extrait d'ovaires et de I'hémolymphe de femelles en vitellogenèse secondaire sont disposés côte à côte. Enfin, l'absorption de l'immunsérum anti-EOV par de l'hémolymphe de femelle en vitellogenèse secondaire supprime toute réaction (fig. 3 ).

L'immunoélectrophorèse croisée, réalisée avec le même immunsérum que précédemment, confirme l'hétérogénéité de la vitellogénine et des vitellines (fig. 4 et 5). Le nombre de systèmes précipitants se révèle particulièrement élevé pour les extraits d'ovaires en vitellogenèse secondaire (5 à 6). Avec cette technique également, on n'observe de précipitation ni avec l'hémolymphe des mâles, ni avec l'hémolymphe de femelles en repos sexuel. Lorsque l'immunsérum anti-EOV est absorbé par de l'hémolymphe de femelle en vitellogenèse secondaire, plus aucune réaction n'est décelable.

Incorporation de la leucine tritiée dans les protéines sériques des femelles en vitellogenèse secondaire. - Chez les espèces de Crustacés qui ont été étudiées de ce point de vue, notamment l'Amphipode Orchestia gammarellus (Meusy et al., 1974), la synthèse de la vitellogénine constitue un métabolisme particulièrement intense $\left(6 \mathrm{~h}\right.$ après injection de leucine ${ }^{3} \mathrm{H}$, la vitellogénine est, de loin, la protéine sérique la plus fortement marquée).

FIG. 2. - Immunodiffusions mettant en présence: de l'hémolymphe de femelles en vitellogenèse secondaire (1 et 5), de femelles en repos sexuel (3) et de mâle (6), des extraits d'ovaires en vite/logenèse secondaire (EOV) (2 et 4), et un immunsérum anti-EOV (I).

Les lignes de précipitation, dans certains cas légèrement dédoublées, sont en continuité et ne forment pas d'éperon. On note l'absence de réaction avec l'hémolymphe de femelle en repos sexuel (3) et de mâle (6).

FIG. 3. - Immunodiffusions mettant en présence : de l'hémolymphe de femelles en vitellogenèse secondaire $(1,3,4$ et 6$)$, des extraits d'ovaires en vitellogenèse secondaire (EOV) (2 et 5), et un immunsérum anti-EOV normal (I) ou absorbé par de l'hémolymphe de femelles en vitellogenèse secondaire (II).

On remarque que l'absorption supprime toute réaction. L'antigène VG en excès dans l'immunsérum absorbé (HI) réagit avec l'immunsérum non absorbé (I) et donne lieu aux lignes de précipitation observées.

FIG. 4 et 5. - Immunoélectrophorèses croisées en système agarose-agarose d'hémolymphe de femelles en vitellogenèse secondaire (fig. 4) et d'extraits d'ovaires en vitellogenèse secondaire (EOV) (fig. 5). Le gel dans lequel est effectué la seconde migration a été additionné d'un sérum anti-EOV (I).

Ces conditions analytiques confirment l'hétérogénéité de la vitellogénine et des vitellines. 


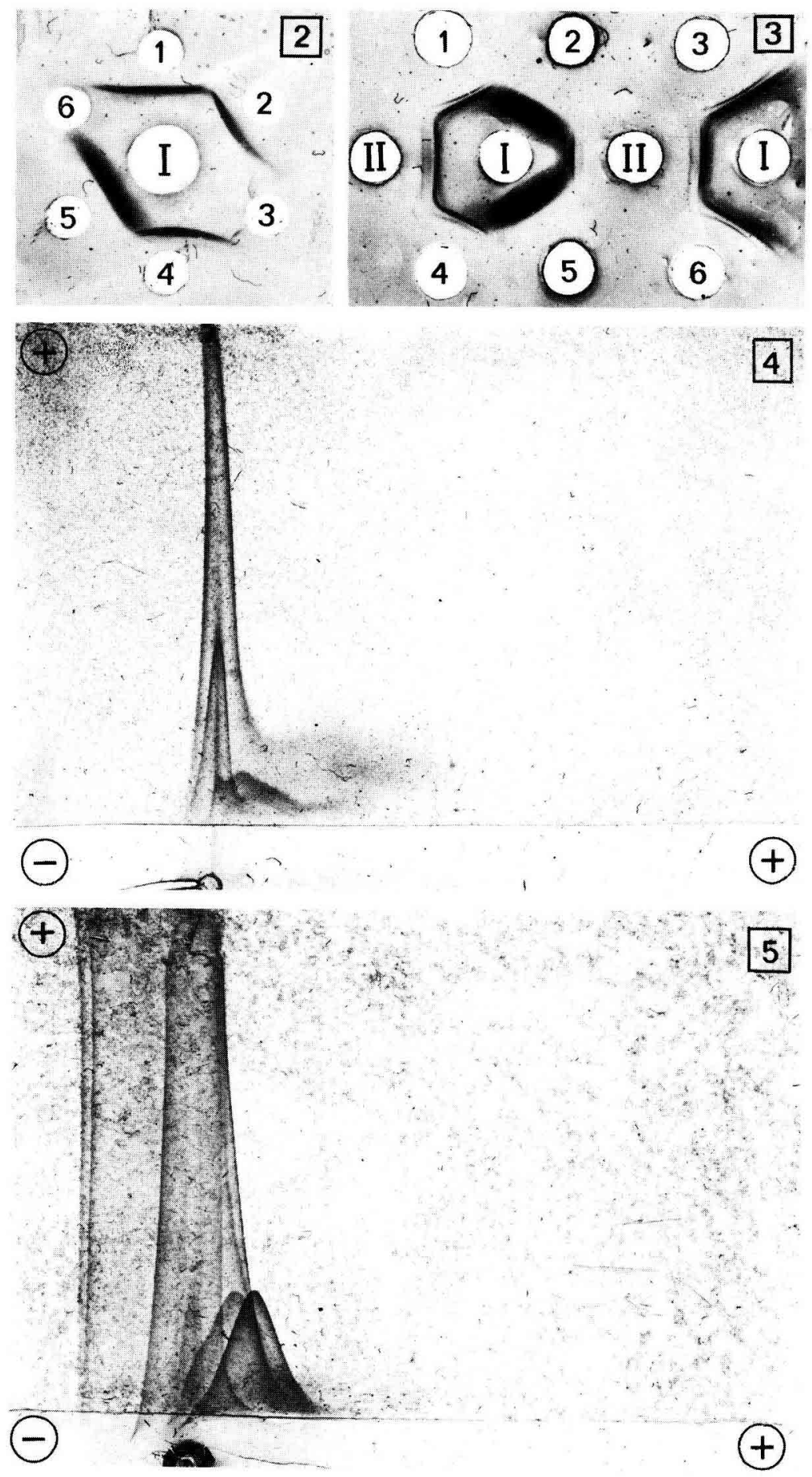


Chez $P$. serratus, les protéines sériques les plus intensément marquées sont la VG 1 et, à un degré beaucoup moindre, la VG 2 (fig. 6). Pour la même durée $d^{\prime}$ incubation, c'est-à-dire $7 \mathrm{~h}$, aucune radioactivité significative $\mathrm{n}^{\prime}$ est détectée au niveau des vitellines ovariennes. Chez les femelles en repos sexuel, aucune incorporation n'est décelable au niveau de VG 1 et VG 2 (fig. 7).

Déclenchement de la synthèse de la vitellogénine chez des femelles en repos sexuel après épédonculation bilatérale. - Quatre femelles de $P$. serratus ont été
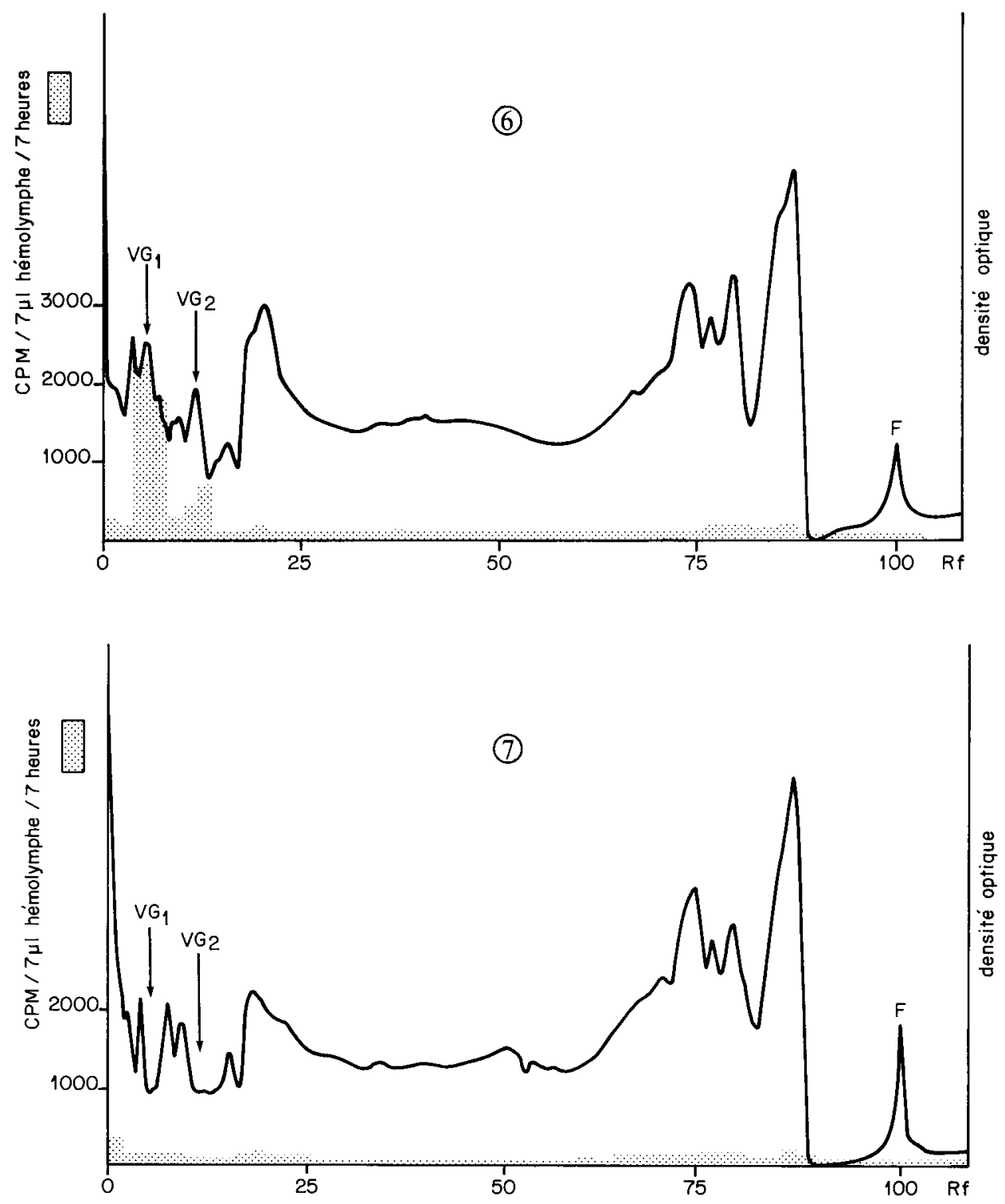


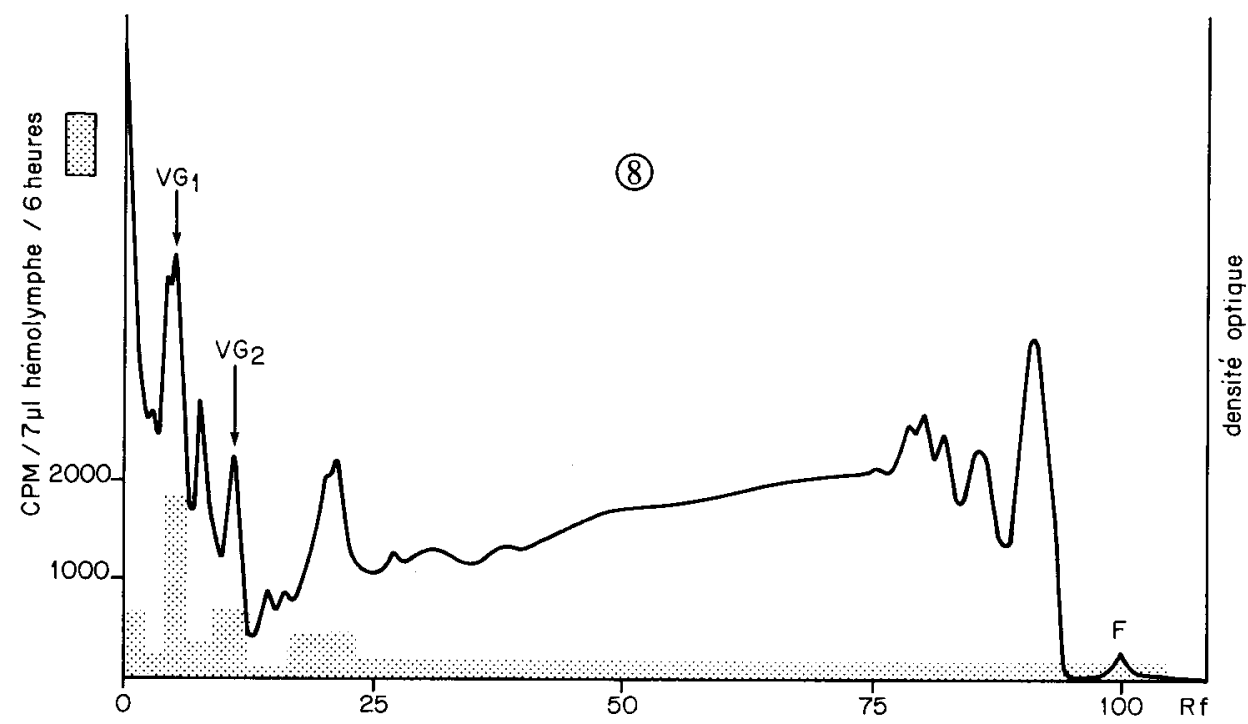

FIG. 6, 7 et 8. - Radioactivité des protéines sériques séparées par électrophorèse, après injection de leucine tritiée à l'animal.

Fig. 6. - Femelle en vitellogenèse secondaire. VG 2 et surtout VG 1 ont incorporé la leucine tritiée.

Fig. 7. - Femelle en repos sexuel. La vitellogénine (VG 1 et VG 2) est absente et aucune incorporation n'est décelée à son niveau.

Fig. 8. - Femelle ayant subi une épédonculation bilatérale pendant la période de repos sexuel (délai post-opératoire : 19 jours).

On remarque la présence de la vitellogénine (VG 1 et VG 2) et l'incorporation du précurseur à son niveau. Comparer avec une femelle en repos sexuel non opérée (fig. 7).

épédonculées bilatéralement en mai, c'est-à-dire durant la période saisonnière de repos sexuel. Nous avons vérifié préalablement que la vitellogénine (VG 1 et VG 2) est absente de l'hémolymphe de ces femelles. Dix neuf jours après l'opération, la vitellogénine est présente (fig. 9). Une injection de leucine ${ }^{3} \mathrm{H}$ à ces animaux expérimentaux permet de mettre en évidence une incorporation dans VG 1 et VG 2 (fig. 8) et par conséquent une synthèse active de vitellogénine.

Ces animaux expérimentaux effectuent la vitellogenèse secondaire; leurs ovaires présentent toutefois une couleur brunâtre fréquente chez les sujets épédonculés, mais inhabituelle chez les femelles normales. On ignore encore les causes de cette anomalie qui a déjà été signalée dans la littérature (Anilkulmar et Adiyodi, 1980). Le vitellus, étudié à l'aide du sérum anti-EOV possède des propriétés immunologiques semblables à celles du vitellus des femelles normales.

Recherche immunohistochimique du lieu de synthèse de la vitellogénine. Sur coupes histologiques de femelles en vitellogenèse secondaire, la réaction immunoenzymatique utilisant le sérum de lapin anti-EOV est fortement positive 


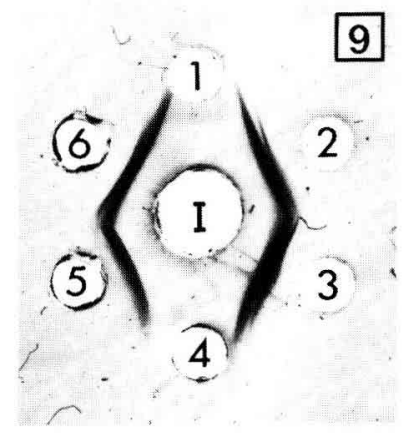

FIG. 9. - Mise en évidence de vitellogénine dans l'hémolymphe, 19 jours après épédonculation bilatérale de femelles en repos sexuel (2 et 5).

A titre de témoins : hémolymphe de femelles en repos sexuel non opérées (1 et 4) et de femelles en vitellogenèse secondaire ( 3 et 6 ). Au centre (I), sérum anti-extraits d'ovaires en vitellogenèse secondaire anti-EOV.

au niveau du tissu adipeux sous-épidermique latéral et surtout ventral (fig. 10). Une certaine réactivité peut aussi être observée au niveau de l'épiderme.

Le tissu adipeux profond est faiblement coloré par la réaction. Hormis l'ovaire (fig. 13) et l'hémolymphe accumulée dans les lacunes, les autres organes et tissus donnent une réaction négative.

Lorsque l'immunsérum est remplacé par du sérum de lapin témoin (fig. 11 et 14), ou lorsqu'il est absorbé par un extrait de femelle en vitellogenèse, aucune réaction n'est observée.

Le tissu adipeux sous-épidermique des témoins physiologiques - mâles et femelles en repos sexuel (fig. 12) - ne réagit pas avec l'immunsérum.

Mise en évidence de vitellines dans des ovaires de femelles en repos sexuel. - Bien que, par définition, les ovaires des femelles en repos sexuel n'effectuent

FIG. 10 et 13. - Localisation de la vitellogénine au niveau du tissu adipeux sous-épidermique et de l'ovaire d'une femelle en vitellogenèse secondaire par la méthode immunoenzymatique d'Avraméas (1969) (voir « Matériel et Méthodes »). L'immunsérum spécifique utilisé est dirigé contre les vitellines (anti-EOV). Coupe transversale de I'animal au niveau du céphalothorax.

cnv : chaîne nerveuse ventrale ; ct : cuticule ; ep : épiderme ; $m$ : muscles ; ov : ovaire ; tase : tıssu adipeux sous-épidermique.

FIG. 11 et 14. - L'antisérum spécifique a été remplacé par du sérum prélevé avant immunisation (témoin).

Aucune réaction n'est décelable.

FIG. 12. - Coupe transversale de femelle en repos sexuel traitée par le sérum anti-vitellines (anti-EOV).

Le tissu adipeux sous-épidermique ventral donne une réaction négative. 

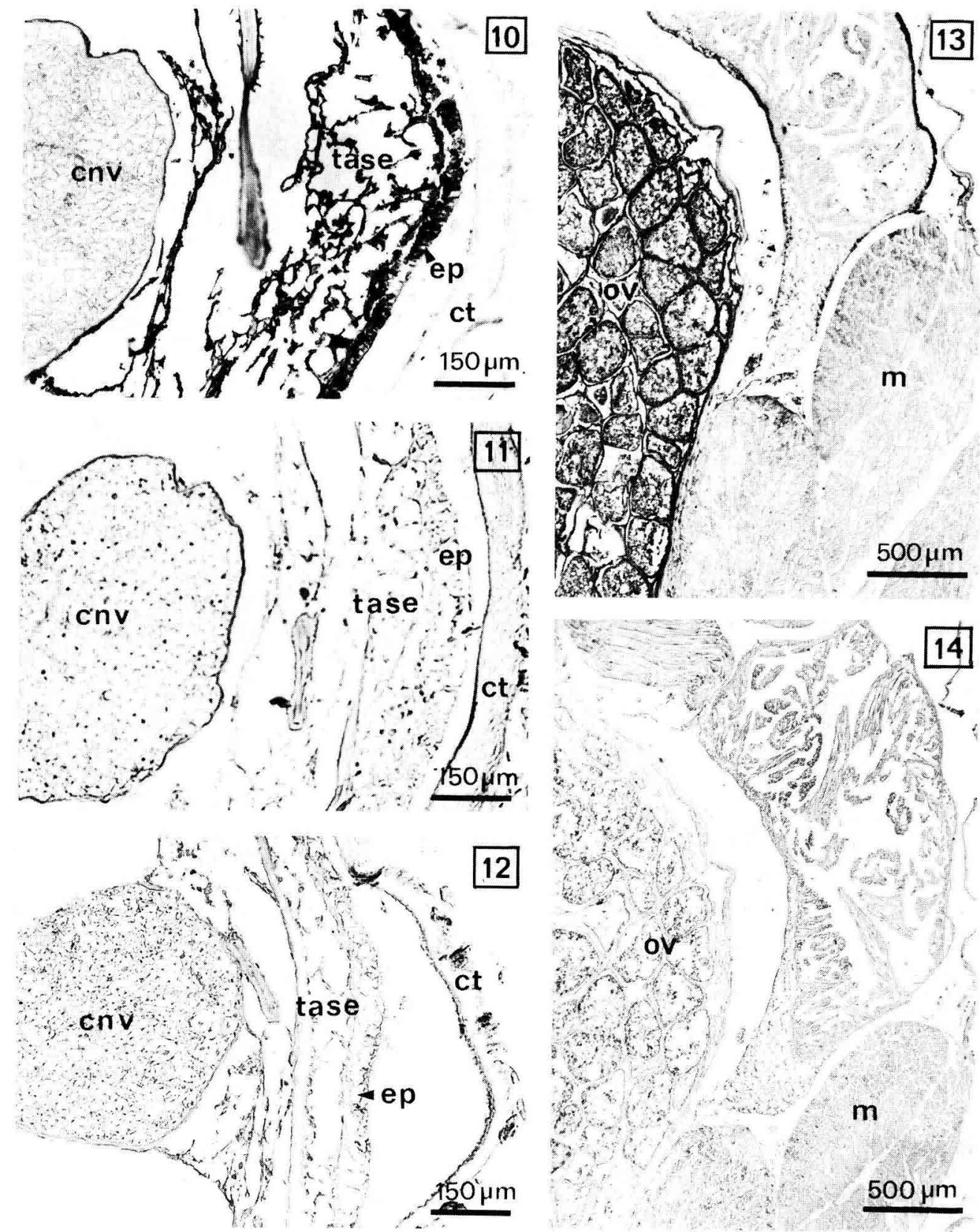

m

$500 \mu \mathrm{m}$ 
pas de vitellogenèse secondaire, des traces de matériel réagissant avec le sérum anti-EOV ont parfois été détectées. Dans le cas présenté dans la figure 15 , le volume des extraits d'ovaires «en repos » soumis à la double-diffusion contre le sérum anti-EOV est 10 fois supérieur à celui de l'extrait d'ovaires en début de vitellogenèse secondaire (couleur vert pâle) préparé dans les mêmes conditions. L'emplacement du matériel précipité indique néanmoins un excès d'anticorps. Par conséquent, ces ovaires de femelles en repos sexuel contiennent une quantité de vitellines plus de 10 fois inférieure à celle qui est présente dans les ovaires en début de vitellogenèse secondaire. Dans d'autres cas, les vitellines sont en quantité encore plus faibles ou ne sont pas décelables.

II faut noter que la ligne de précipitation, légèrement dédoublée, fournie par l'extrait d'ovaires de femelles en repos sexuel et les lignes de précipitation dues à I'hémolymphe et à l'extrait d'ovaires de femelles en vitellogenèse secondaire ne forment aucun éperon entre elles.

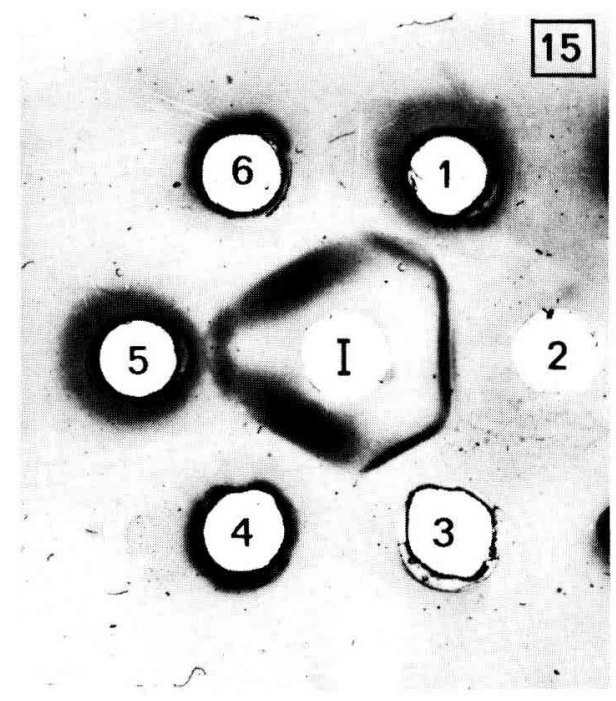

FIG. 15. - Mise en évidence de traces de vitellines dans les ovaires de femelles en repos sexuel. 1 et 5 : extraits d'ovaires de femelles en repos sexuel ; 2, 4 et $6:$ hémolymphe de femelles en vitellogenèse secondaire ; 3 : extrait d'ovaire en début de vitellogenèse secondaire (EOV) : 1 : sérum anti-EOV (dirigé exclusivement contre les vitellines).

On remarque que les précipités, légèrement dédoublés, formés entre 1-I d'une part et entre 2-I $d^{\prime}$ autre part, ne forment aucun éperon entre eux. Le volume des extraits d'ovaires de femelles en repos sexuel déposé en 1 et 5 est 10 fois supérieur à celui de l'extrait d'ovaire en vitellogenèse secondaire déposé en 3.

\section{Discussion et conclusions.}

Les techniques électrophorétiques et immunologiques montrent qu'il existe chez les femelles en vitellogenèse secondaire de Palaemon serratus un précurseur 
sérique du vitellus, la vitellogénine, synthétisé hors de l'ovaire et immunologiquement indistinguable des vitellines.

II importe de noter que :

- le sérum anti-EOV est complètement dépourvu d'anticorps précipitant avec I'hémolymphe des femelles en repos sexuel ou avec celle des mâles. II est donc exempt, en particulier, d'anticorps dirigés contre des protéines sériques éventuellement présentes dans les EOV.

- I'absorption du sérum anti-EOV par de l'hémolymphe de femelles en vitellogenèse secondaire supprime toute réaction ultérieure de ce sérum tant avec cette hémolymphe qu'avec un extrait d'ovaires en vitellogenèse secondaire (EOV). Ce résultat indique que l'immunsérum ne comporte pas d'anticorps dirigés contre des protéines ovariennes autres que les vitellines - par exemple contre le vitellus primaire - et est spécifique du système vitellogénine-vitellines.

- la ou les lignes de précipitation formées en double-diffusion par l'hémolymphe de femelles en vitellogenèse secondaire et par les extraits d'ovaires en vitellogenèse secondaire (EOV) contre le sérum anti-EOV se raccordent complètement et ne forment jamais d'éperon. Aucune différence immunologique entre vitellines et vitellogénine ne peut donc être mise en évidence.

-7 heures après l'injection de leucine ${ }^{3} \mathrm{H}$ à des femelles en vitellogenèse secondaire, on observe un marquage des 2 bandes qui correspondent, sur les électrophorèses d'hémolymphe, à la vitellogénine, alors que les vitellines ne présentent pas encore de marquage significatif. Les constituants de ces 2 bandes ne sont donc pas des produits de résorption du vitellus, mais constituent au contraire un précurseur d'origine extra-ovarienne du vitellus. Ajoutons qu'en période de reproduction les phénomènes de résorption n'interviennent qu'occasionnellement, en particulier après une ponte incomplète.

L'étude immunohistologique permet de montrer de façon très évidente une forte concentration de vitellogénine au niveau du tissu adipeux sous-épidermique latéral et surtout ventral chez les femelles en vitellogenèse secondaire. La réaction parfois observée au niveau de l'épiderme contigu est probablement due à la diffusion de la vitellogénine au cours de la fixation. Les autres organes ou tissus, ovaires et hémolymphe exceptés, n'étant pas immunoréactifs, il ne fait guère de doute que le tissu adipeux sous-épidermique synthétise la vitellogénine, comme c'est le cas chez l'Amphipode $O$. gammarellus (Junéra et Croisille, 1980 ; Croisille et Junéra, 1980) et les Isopodes Porcellio dilatatus (Picaud, 1980) et Idotea balthica basteri (Souty et Picaud, 1981). Sa dispersion et son faible développement rendent malheureusement l'extraction et l'incubation in vitro de ce tissu, difficilement réalisables.

La vitellogénine et les vitellines ne paraissent pas être des substances homogènes et/ou stables. Les double-diffusions effectuées contre le sérum anti-EOV donnent généralement lieu à 2 , parfois 3 , lignes de précipitation et confirment les résultats de l'électrophorèse. En immunoélectrophorèse croisée contre le même immunsérum, le nombre des systèmes précipitants apparaît encore plus élevé. Cette hétérogénéité n'est pas une particularité propre au système vitellogénine/vitellines de $P$. serratus. En effet, nous avons montré que le vitellus d'Orchestia gammarellus présente après électrophorèse 2 vitellines (lipovitellines) 
immunologiquement indistinguables et pouvant se transformer l'une en l'autre, mais de poids moléculaire diffèrent (Junéra et al., 1977 ; Meusy, 1980).

Chez les femelles en repos sexuel où la vitellogénine est absente de I'hémolymphe, la faible ligne de précipitation parfois obtenue en double-diffusion avec les extraits d'ovaires est un résultat inattendu. La technique immunohistochimique a permis de localiser ces vitellines à la périphérie de l'ovaire, 1) dans $d^{\prime}$ anciens ovocytes non pondus et incomplètement résorbés, 2) dans des ovocytes considérés comme bloqués en fin de vitellogenèse primaire (résultats non publiés). Ces derniers ont probablement effectué un tout début de vitellogenèse secondaire lorsque sont intervenus la période de repos sexuel et l'arrêt de la synthèse de la vitellogénine.

II est probable que l'arrêt de la période de reproduction est dû à l'intervention de l'hormone gonado-inhibitrice $(\mathrm{GIH})$ secrétée par le complexe organe $X$ glande du sinus localisé dans les pédoncules oculaires. La mise en évidence du déclenchement de la synthèse de la VG chez les femelles en repos sexuel par épédonculation bilatérale constitue à cet égard un résultat important. Toutefois, nous ignorons à quel(s) niveau(x) agirait cette hormone. Ses différentes cibles pourraient être :

- le tissu adipeux sous-épidermique qui cesserait alors de synthétiser la VG ;

- I'ovaire lui-même qui ne secréterait plus la VSOH (Vitellogenin Stimulating Ovarian Hormone : Junéra et al., 1977), amenant ainsi I'arrêt de la synthèse de la VG :

- la membrane plasmique des ovocytes qui ne pourraient plus prélever la VG circulante.

La mise en évidence chez $P$. serratus d'un système vitellogénine-vitellines analogue à celui qui a été décrit chez d'autres espèces de Crustacés n'exclut pas formellement la possibilité d'une origine partiellement ovarienne des vitellines. On sait que tel est le cas chez certains Insectes (Wyss-Huber et Lüscher, 1975 ; Gutzeit, 1980 ; etc...) et que des travaux effectués chez des Crustacés Décapodes ont abouti à des conclusions similaires (Arcier et Bonami chez Palaemon adspersus, 1979 ; Lui, Sage et O'Connor, 1974, Lui et O'Connor, 1976, chez Procambarus ; Lui et O'Connor, 1977, chez Pachygrapsus crassipes). La preuve irréfutable de la participation de l'ovaire à la synthèse des vitellines ne nous paraît pas toutefois avoir été apportée chez les Crustacés. Nous avons appliqué à des ovaires de $P$. serratus le protocole d'incubation et de séparation électrophorétique des protéines ovariennes mis en œuvre par Arcier et Bonami (1979) (travaux non publiés). Après avoir mesuré la radioactivité sur toute la longueur du gel d'acrylamide, nous avons conclu à l'absence d'incorporation significative au niveau des vitellines. De plus, un contrôle histologique des ovaires incubés a révélé des altérations notables qui nous incitent à la prudence. Les travaux de Lui, Sage et O'Connor (1974) et Lui et O'Connor (1976, 1977) appellent également quelques remarques. On peut, en particulier, être surpris par la faible radioactivité de la lipovitelline et de ses sous-unités polypeptidiques qui auraient été synthétisées in vitro. Ainsi, dans leur travail de 1977, Lui et O'Connor, qui ont incubé des ovaires de Pachygrapsus crassipes dans un milieu très riche en leucine marquée $(500$ $\mu \mathrm{Ci}$ pour 5 ou $8 \mathrm{ml}$ de milieu ; $A S=43 \mathrm{Ci} / \mathrm{mmole}$ ) et pendant une longue durée 
$(30 \mathrm{~h}), \mathrm{n}^{\prime}$ ont pourtant détecté qu'une radioactivité -relativement peu importante au niveau de la lipovitelline (un peu moins de $30 \mathrm{dpm} / \mu \mathrm{g}$ de protéine). II serait intéressant d'étudier les synthèses endogènes de l'ovaire en faisant fonctionner I'ARNm extrait des ovocytes et du tissu folliculaire dans un système acellulaire.

En conclusion, il existe chez Palaemon serratus, comme chez l'Amphipode Orchestia gammarellus et les Isopodes, un précurseur extraovarien du vitellus secondaire, la vitellogénine, immunologiquement indistinguable des vitellines. Cette vitellogénine est absente de l'hémolymphe durant la période annuelle de repos sexuel car sa synthèse - au niveau des adipocytes - subit une inhibition due à une neurohormone pédonculaire.

Reçu en octobre 1982.

Accepté en janvier 1983.

Remerciements. - Nous tenons à remercier Monsieur le Professeur $\mathrm{Y}$. Croisille pour ses précieux conseils et pour l'aide amicale qu'il nous a fournie lors de la préparation de l'immunsérum.

\section{Références}

ANILKUMAR G., ADIYODI K. G., 1980. Ovarian growth, induced by eyestalk ablation during the prebreeding season, is not normal in the crab, Paratelphusa hydrodromous (Herbst). Int. J. Invertebr. Reprod., 2, 95-105.

ARCIER J. M., BONAMI J. R., 1979. Contribution à l'étude des lipovitellines chez Palaemon adspersus (Rathke, 1837). Arch. int. Physiol. Biochim., 87, 471-484.

ARCIER J. M., TOURNAMILLE J., 1974. Recherches immunochimiques sur les protéines de vitellogenèse chez Palaemon adspersus (Rathke, 1837) (Crustacés, Décapodes, Natantia). C.R. Acad. Sci. Paris, sér. D, 278, 495-498.

AVRAMEAS S., 1969. Indirect immunoenzyme techniques for the intracellular detection of antigens. Immunochemistry, 6, 825-831.

BEAMS H. W., KESSEL R. G., 1962. Intracisternal granules of endoplasmic reticulum in the crayfish oocyte. J. Cell Biol., 13, 158-162.

BEAMS H. W., KESSEL R. G., 1963. Electron microscope studies on developing crayfish oocytes with special reference to the origin of yolk. J. Cell Biol., 18, 621-650.

CHARNIAUX-COTTON H., 1978. L'ovogenèse, la vitellogénine et leur contrôle chez le Crustacé Amphipode Orchestia gammarellus (Pallas). Comparaison avec d'autres Malacostracés. Arch. Zool. exp. Gen., 119, 365-397.

CROISILLE Y., JUNÉRA H., 1980. Recherche du lieu de synthèse de la vitellogénine chez le Crustacé Amphipode Orchestia gammarellus (Pallas). Démonstration, à l'aide d'anticorps spécifiques, de la présence de vitellogénine dans le tissu adipeux sous-épidermique des femelles en vitellogenèse secondaire. C.R. Acad. Sci. Paris, sér. D, 290, 1487-1490.

DAVIS B. J., 1964. Disc-électrophoresis. II. Method and application to human serum proteins. Ann. N.Y. Acad. Sci., 121, 404-427.

FYFFE W. E., O'CONNOR J. D., 1974. Characterization and quantification of a crustacean lipovitellin. Comp. Biochem. Physiol., 47B, 851-867.

GANION L. R., KESSEL R. G., 1972. Intracellular synthesis, transport, and packing of proteinaceous yolk in oocytes of Orconectes immunis. J. Cell Biol., 52, 420-437.

GRAHAM R. C., KARNOVSKY M. J., 1966. The early stages of absorption of injected horseradish peroxidase into the proximal tubules of mouse kidney. Ultrastructural cytochemistry by a new technique. J. Histochem. Cytochem., 14, 291-302. 
GUTZEIT H. O., 1980. Yolk synthesis in ovarian follicles of Drosophila. Wilhelm Roux's Archives, 189, $221-224$.

JUNÉRA H., CROISILLE Y., 1980. Recherche du lieu de synthèse de la vitellogénine chez le Crustacé Amphipode Orchestia gammare/lus (Pallas). Mise en évidence d'une activation de la synthèse protéique dans le tissu adipeux sous-épidermique en liaison avec la production de vitellogénine. C.R. Acad. Sci. Paris, sér. D, 290, 703-706.

JUNÉRA H., MARTIN M., SOLARI A., MEUSY J. J., 1977. Détermination du poids moléculaire de la vitellogénine et des lipovitellines d'Orchestia gammarellus, Crustacé Amphipode. C.R. Acad. Sci. Paris, sér. D, 285, 909-912.

KESSEL R. G., 1968. Mechanisms of protein yolk synthesis and deposition in crustacean oocytes. Z. Zelfforsch., 89, 17-38.

LUI C. W., O'CONNOR J. D., 1976. Biosynthesis of lipovitelin by the crustacean ovary. II. Characterization of an in vitro incorporation of amino acids into the purified subunits. $J$. exp. Zool., 195, 41-52.

LUI C. W., O'CONNOR J. D., 1977. Biosynthesis of crustacean lipovitellin. III. The incorporation of labeled amino acids into the purified lipovitellin of the crab Pachygrapsus crassipes. J. exp. Zool., 199, 105-108.

LUI C. W., SAGE B. A., O'CONNOR J. D., 1974. Biosynthesis of lipovitellin by the crustacean ovary. J. exp. Zool., 188, 289-296.

MEUSY J. J., 1980. Vitellogenin, the extraovarian precursor of the protein yolk in Crustacea : a review. Reprod. Nutr. Dévelop., 20, 1-21.

MEUSY J. J., JUNÉRA H., CROISILLE Y., 1974. Données sur la synthèse de la fraction protéique femelle chez Orchestia gammarel/us (Pallas) (Crustacé Amphipode), au cours de l'intermue et chez les femelles en repos sexuel. C.R. Acad. Sci. Paris, sér. D, 279, 587-590.

OUCHTERLONY O., 1948. Antigen antibody reactions in gels. Arkiv. Kemi. Mineral. Geol., $26 \mathrm{~B}, \mathrm{n}^{\circ} 14$.

PICAUD J. L., 1980. Vitellogenin synthesis by the fat body of Porcellio dilatatus Brandt (Crustacea, Isopoda). Int. .. Invertebr. Reprod., 2, 341-349.

PICAUD J. L., SOUTY C., 1980a. Démonstration immunohistochimique de la présence de vitellogénine dans le tissu adipeux et l'hépatopancréas du Crustacé Isopode Oniscoïde Porcellio dilatatus (Brandt). C.R. Acad. Sci. Paris, sér. D, 290, 123-125.

PICAUD J. L., SOUTY C., 1980b. Démonstration par immunoautoradiographie de la synthèse de la vitellogénine par le tissu adipeux de Porcellio dilatatus Brandt (Crustacé, Isopode). C.R. Acad. Sci. Paris, sér. D, 290, 1019-1021.

SOUTY C., PICAUD J. L., 1981. Vitellogenin synthesis in the fat body of the marine crustacean Isopoda, Idotea balthica basteri, during vitellogenesis. Reprod. Nutr. Dévelop., 21, 95-101.

VAGO C., QUIOT J.-M., 1969. Recherches sur la composition des milieux pour culture de cellules d'Invertébrés. Ann. Zool. Ecol. anim., 1, 281-288.

WYSS-HUBER M., LÜSCHER M., 1975. Protein synthesis in "fat body " and ovary of the physogastric queen of Macrotermes subhyalinus. J. Insect. Physiol., 21, 1697-1704. 